2018 IEEE 9th Annual Information Technology, Electronics and Mobile Communication Conference, IEMCON 201816 January 2019, Article number 8615063, Pages 328-3349th IEEE Annual Information Technology, Electronics and Mobile Communication Conference, IEMCON 2018; Vancouver; Canada; 1 November 2018 through 3 November 2018; Category numberCFP1879Z-ART; Code 144366

\title{
Cloud Model for Purchase Management in Health Sector of Peru based on IoT and Blockchain(Conference Paper)
}

- Celiz, R.C. ${ }^{\mathrm{E} E m a i l}$ Author,

- De La Cruz, Y.E.aEmail Author,

- Sanchez, D.M. ${ }^{\circ}$ Email Author

- aFaculty of Engineering, Peruvian University of Applied Sciences, Lima, Peru

- ${ }^{\circ}$ Computer Science Peruvian, University of Applied Sciences, Lima, Peru

Abstract_View references (29)

Purchase management of medical supplies is a critical and important process that affects the services provision quality. Nonetheless, it is facing a growing pressure to provide visibility and traceability of the purchase, to reduce fraud, to improve flexibility and to ensure communication between everyone involved. Currently, private health institutions in Peru choose to implant different software products within the same company with restricted visibility access to other concerned parties and based on information from a single source. A new alternative is Blockchain technology, since it provides a single source of shared truth to all participants and ensures that the information cannot be altered, thus offering high levels of transparency that, together with loT technology, creates not only visibility about where things are, but also traceability, showing the current state of things. (C) 2018 IEEE.

Author keywords

BlockchainHealth SectorloTPharmaceuticsPurchasingTraceabilityVisibility

Indexed keywords

Engineering controlled terms: BlockchainHealthMobile telecommunication systemsPurchasingSalesVisi 
Engineering uncontrolled terms

Engineering main heading: Internet of things

- ISBN: 978-153867266-2

- Source Type: Conference Proceeding

- Original language: English

- DOI: 10.1109/IEMCON.2018.8615063

- Document Type: Conference Paper

- Volume Editors: Chakrabarti S.,Saha H.N.

- Sponsors: IEEE Vancouver Section,Institute of Engineering and Management (IEM),UBC,University of Engineering and Management (UEM)

- Publisher: Institute of Electrical and Electronics Engineers Inc. 